\title{
Immunomodulation as Therapy for Fungal Infection: Are We Closer?
}

\author{
Qi Hui Sam 1,2,3, Wen Shan Yew ${ }^{2,3}$, Chaminda J. Seneviratne ${ }^{4}$, Matthew Wook Chang ${ }^{2,3}$ \\ and Louis Yi Ann Chai ${ }^{1,3,5,6 *}$ \\ 1 Division of Infectious Diseases, University Medicine Cluster - National University Health System, Singapore, Singapore, \\ 2 Department of Biochemistry, Yong Loo Lin School of Medicine, National University of Singapore, Singapore, Singapore, \\ ${ }^{3}$ Synthetic Biology for Clinical and Technological Innovation (SynCTI), Life Sciences Institute, National University \\ of Singapore, Singapore, Singapore, ${ }^{4}$ Oral Sciences, Faculty of Dentistry, National University of Singapore, Singapore, \\ Singapore, ${ }^{5}$ Department of Medicine, Yong Loo Lin School of Medicine, National University of Singapore, Singapore, \\ Singapore, ${ }^{6}$ National University Cancer Institute, Singapore, Singapore
}

Invasive fungal disease (IFD) causes significant morbidity in immunocompromised patients due to their weakened immune system. Immunomodulatory therapy, in synergy with existing antifungal therapy, is an attractive option to enhance their immune system and aid clearance of these opportunistic pathogens. From a scientific and clinical perspective, we explore the immunotherapeutic options to augment standard antifungal drugs for patients with an IFD. We discuss the range of immunomodulatory therapies being considered in IFD - from cytokines, including G-CSF, GM-CSF, M-CSF, IFN$\gamma$, and cytokine agonists, to cellular therapies, consisting of granulocyte transfusion, adoptive T-cell, CAR T-cell, natural killer cell therapies, and monoclonal antibodies. Adjunct pharmaceutical agents which augment the immunity are also being considered. Lastly, we explore the likelihood of the use of probiotics and manipulation of the microbiome/mycobiome to enhance IFD treatment outcomes.

Keywords: immunotherapy, mycosis, invasive fungal disease, aspergillosis, candidiasis, anti-fungal, microbiome, mycobiome

\section{INTRODUCTION}

For many people living with cancer, rheumatological diseases and, solid organ and stem cell transplant recipients, medical advances have allowed them to prolong their lives. However, many of the treatments involve the alteration or weakening of the patient's immune system; this predisposes the immunosuppressed patient to infections by opportunistic pathogens. Of the opportunistic infections, one of the most problematic is fungal infections. The disease spectrum of IFDs encompasses candidiasis, aspergillosis, cryptococcosis, zygomycosis, penicilliosis, histoplasmosis, and infections by other fungi such as, Scedoporium, Fusarium, and Sporothrix species. IFDs cause significant morbidity and mortality among immunocompromised patients, ranging between $30 \%$ and more than $60 \%$ in susceptible patients (Neofytos et al., 2009). In a review of the burden of

Abbreviations: AdV, adenovirus; AML, acute myelogenous leukemia; atRA, all-trans retinoic acid; BKV, BK virus; CAR, chimeric antigen receptor; CMV, cytomegalovirus; CSF, cerebrospinal fluid; EBV, Epstein-Barr virus; FDA, U.S. Food and Drug Administration; FN, febrile neutropenia; G-CSF, granulocyte colony-stimulating factor; GM-CSF, granulocytemacrophage colony-stimulating factor; GTX, granulocyte transfusion; GVHD, graft vs. host disease; HHV-6, human herpesvirus-6; HSCT, hematopoietic stem cell transplant; IFD, invasive fungal disease; IFN- $\gamma$, interferon-gamma; IRIS, immune reconstitution inflammatory syndrome; mAbs, monoclonal antibodies; M-CSF, macrophage colony-stimulating factor; MHC, major histocompatibility complex; NK cell, natural killer cell; RCTs, randomized controlled trials; SOCS3, suppressor of cytokine signaling 3; VZV, Varicella-Zoster virus. 
fungal disease in Europe, the overall mortality in patients with IFD averaged around 35 to $45 \%$, and be as high as $82.4 \%$ (Drgona et al., 2014). Globally, various international centers have yielded similarly high mortality rates from fungal infections (Brown et al., 2012; Rajasingham et al., 2017). The persistence of these high mortality rates, despite antifungal treatment, is the consequence of the immune predisposition or compromised status of the host to respond adequately to infection. The limited options of antifungal therapeutics available highlight the need to develop novel therapeutic strategies to optimize patient outcomes.

The strategy required to fight against fungal infections can be likened to playing battles in an electronic game, for example, battling other pokemon in Pokémon Go. To win a battle where you face a strong opponent, you either strengthen yourself, weaken your opponent, or do both. Antifungals are our potions to weaken the invasive fungi. Like humans, fungi are eukaryotic, making the hunt for fungal-specific targets more complicated, with many antifungals causing significant adverse side effects to the patient. The other way to win is by augmenting the host immunity; to continue the analogy, immunotherapy is our potion to strengthen ourselves to clear the infection. For an immunocompromised patient with an IFD, immunotherapy becomes an attractive option to add the immunity back. Immunomodulatory agents either turn up the immune response in the case of immunosuppression, or turn it down to prevent further harm to the patient in the case of an overactive immune system.

In this manuscript, we will review immunotherapies currently in use in the context of fungal infections, as well as other potential immunomodulatory modalities at their various stages of development.

\section{CYTOKINE THERAPY}

\section{Granulocyte Colony-Stimulating Factor (G-CSF) and Granulocyte-Macrophage Colony-Stimulating Factor (GM-CSF)}

Granulocyte colony-stimulating factor, generic names include filgrastim and lenograstim, primarily stimulates neutrophil production and maturation. Chemotherapy may be myelosuppressive, causing neutropenia, and G-CSF can be used adjunctly to restore neutrophil counts (reviewed in Wright et al., 2017).

Granulocyte-macrophage colony-stimulating factor, generic names include sargramostim and molgramostim, is an important immunomodulatory cytokine. There is a large amount of literature about its functions, as it stimulates a wider range of immune cells than G-CSF. In brief, GM-CSF stimulates maturation of dendritic cells from monocyte precursors, differentiation of macrophages, and proliferation and activation of macrophages, monocytes, neutrophils, eosinophils, dendritic cells, and microglia (reviewed in Hamilton, 2008; van de Veerdonk et al., 2012; Shiomi and Usui, 2015; Scriven et al., 2017). In GM-CSF deficient mice, bacterial and viral clearance by alveolar macrophages is impaired, suggesting its role in aiding pathogen clearance (LeVine et al., 1999; Paine et al., 2000; Berclaz et al., 2002).

Neither G-CSF nor GM-CSF are directly linked to improvement in IFD-related incidence and mortality, but they may aid the faster recovery of the patient. During chemotherapy-induced FN, G-CSF is commonly prescribed. G-CSF reduces the duration of neutropenia and the length of stay during FN episodes; however, its administration did not conclusively improve infection-related mortality in FN (Clark et al., 2005). The latest Cochrane Database Systematic Review on the use of colony-stimulating factor in FN (both G-CSF and GM-CSF), reflected similar findings when they compared the results of 14 RCTs (Mhaskar et al., 2014). While the paper concluded there was a shorter duration of neutropenia, faster recovery of fever, and shorter empiric antibiotic use, the administration of the colony-stimulating factors did not improve overall mortality and infection, including fungal-related mortality. In a phase IV randomized clinical trial comparing the effect of prophylactic treatment of 206 allogenic HSCT recipients with G-CSF, GM-CSF, or both G-CSF + GM-CSF, Wan et al. (2015) found IFD-related mortality after 600 days to be lower in the GM-CSF, G-CSF + GM-CSF groups as compared to G-CSF-alone group; $1.47,1.45$, and $11.59 \%$, respectively $(P=0.016)$. The results of this trial may point in the direction of further studies with prophylactic GM-CSF, with or without G-CSF, to reduce IFD-related mortality in patients. Regarding IFD incidence, Hovi et al. (2000) and Wan et al. (2015) found no difference when G-CSF or GM-CSF were used with antifungals prophylactically in allogenic or autologous HSCT patients.

On an individual patient level, GM-CSF has shown promising results. Chen et al. (2017) reported success where GM-CSF was used as adjunct therapy to treat Aspergillus ventriculitis. GMCSF was given in conjunction with voriconazole, amphotericin $\mathrm{B}$, and caspofungin. After 2 years of therapy, the patient fully recovered and remained in remission. The role of GM-CSF in this case, was deemed significant by the authors, as fungal ventriculitis has a high mortality of $67 \%$ with conventional treatment. In a case of Scedosporium apiospermum with sub-optimal response to voriconazole, success was reported after switching to the antifungal micafungin co-administered with GM-CSF (Goldman et al., 2016).

The data above supports consideration for the use of G-CSF and GM-CSF, which constitutes our most familiar practice of immunotherapy, in particular G-CSF at the bedside. Concerns regarding thromboembolic events with colony-stimulating factor use are not well-founded based on findings of the Cochrane Review (Mhaskar et al., 2014). Perceived gains, however, are secondary until there can be firmer primary outcome data, such as reduction in infection or IFD-attributable mortality.

\section{Macrophage Colony-Stimulating Factor (M-CSF)}

Macrophage colony-stimulating factor (also known as CSF-1), unlike G-CSF and GM-CSF, is not approved by the FDA for use in patients. M-CSF, as its name suggests, mainly promotes the growth of macrophages (reviewed in Hume and MacDonald, 2012). Kandalla et al. (2016) treated 
transplant-mouse models with M-CSF and found improved survival of the mice when challenged with Aspergillus fumigatus, from $10 \%$ in controls to $60 \%$ in M-CSF treated mice. Correspondingly, fungal burden in the lung, liver, and heart was lower in the M-CSF treated mice. Kandalla et al. (2016) found that M-CSF induced myeloid commitment of HSCs but not G-CSF. In the case series described by Nemunaitis et al. (1993) (reviewed in Hume and MacDonald, 2012), 46 stem cell transplantation patients with IFD were given recombinant human M-CSF with conventional antifungal treatment; there was better survival in the patients who received M-CSF, as compared to historical controls ( $27 \%$ vs. $5 \%, P=0.027)$. There has been no follow up to these reported findings. In cancer, macrophages constitute both the tumor and tumor microenvironment, existing as tumor-associated macrophages, and can represent up to $50 \%$ of the tumor cell mass (reviewed in Medina-Echeverz et al., 2014). In this respect, the administration of M-CSF raises concerns of its theoretical predisposition to accelerate disease progression in cancer patients by enhancing this macrophage population. Hence, M-CSF, though it sounds like a cousin of the G-CSF and GM-CSF, and a potential therapeutic agent, might not be appropriate for the use upfront in cancer patients against fungal infections.

\section{Interferon-Gamma (IFN- $\gamma$ )}

Recombinant IFN- $\gamma$, also known as $\operatorname{rIFN}-\gamma 1 \mathrm{~b}$, was approved by the US FDA in 2000 for the use in prophylaxis against infections for patients with chronic granulomatous disease, and to delay the progression of severe osteopetrosis (FDA, 2000). With greater understanding of the action of IFN- $\gamma$ in fungal infections, some groups which have trialed IFN- $\gamma$ as adjunctive therapy for patients with severe IFD have had encouraging results: in two patients with chronic pulmonary aspergillosis (Kelleher et al., 2006), in an HIV positive and two HIV-negative patients with pulmonary aspergillosis, when used in conjunction with GM-CSF (Bandera et al., 2008), seven kidney transplant patients with IFD (Armstrong-James et al., 2010), two cases of cerebral aspergillosis (Estrada et al., 2012; Mezidi et al., 2014), and a pediatric patient with disseminated candidiasis (Buddingh et al., 2015). All these case reports described recovery of the patients. Taking into account the likely positive publication bias, further clinical trials are vital to ascertain its efficacy.

There have been a few clinical trials for the use of IFN- $\gamma$ in IFD. Pappas et al. (2004) conducted a phase 2, doubleblind, placebo-controlled trial in HIV positive patients with acute Cryptococcus meningitis. The study showed a trend toward a more rapid sterilization of CSF in the patients who received IFN- $\gamma$ compared to the placebo $(P=0.072)$. Their study also showed that the lower dosage of $100 \mu \mathrm{g}$ of IFN- $\gamma$ was sufficient to achieve similar results compared to the $200 \mu \mathrm{g}$ recipients. IFN- $\gamma$ was also well tolerated among the patients, with few significant adverse events. Another open label RCT compared the addition of IFN- $\gamma$ to standard amphotericin B therapy in HIV positive patients with Cryptococcus meningitis (Jarvis et al., 2012). The study found that short-course IFN $\gamma$ therapy significantly increased the rate of CSF Cryptococcus clearance $(P=0.02)$, with no significant increase in adverse events. In addition, they found that two doses of IFN- $\gamma$ were as effective as six doses. Taken together, these two trials demonstrated that two doses of $100 \mu \mathrm{g}$ of IFN- $\gamma$ was sufficient for the faster clearance of fungal infection. Beyond Cryptococcosis, Delsing et al. (2014) conducted a clinical series between 2010 and 2013, using IFN- $\gamma$ with standard antifungal therapy, the trial had to be terminated early due to low enrollment. The study recruited eight patients with invasive Candida and/or Aspergillus infections. The results were encouraging in that five of the eight patients treated with IFN- $\gamma$ recovered from the IFD. Two of the ICU patients included in the study died due to infectious complications. One of the eight patients was lost to follow up. The authors observed that IFN- $\gamma$ elevated IL-17 and IL-22 production. Monocyte and lymphocyte numbers also significantly increased after the initiation of IFN- $\gamma$ therapy.

Numerous studies have produced promising results in the use of IFN- $\gamma$ treatment, and encouraging results from the phase II studies discussed above, point to IFN- $\gamma$ as a potential adjunct to standard treatment. Further larger clinical trials have to be conducted to establish the therapy's efficacy and conditions for use.

\section{Cytokine Agonist Therapy}

The capacity of the host immunity to recognize and respond to the fungal pathogen is mediated by a range of pathogen recognition receptors including Toll-like receptors (TLRs), such as TLRs 2, 4, 7, 9, and C-type lectin receptors (CLRs), e.g., Dectin1. Accounts of the signaling pathways triggered upon recognition of the fungal ligand and the consequent host inflammatory responses elicited to date have been well-described by many esteemed publications (Gow et al., 2011; Romani, 2011; Wüthrich et al., 2012). All these constitute the host innate and adaptive immune arms against infection. Naturally, modulation and manipulation of these signaling pathways alter the net immune response and may alter the outcome of infection.

A modality considered was the use of an antigen or adjuvant to augment the inflammatory response. Various fungal-originated moieties may be utilized as antigens to activate the host Th1, Treg or Th17 cytokine arms, perceived to confer protection against infection. Examples of fungal antigens include Pep1p, a secreted fungal aspartic protease, and the various glycosylphosphatidylinositol (GPI)-anchored proteins of the fungal cell wall, as demonstrated in mice model studies (Bozza et al., 2009). The administration of cytosine guanine oligodeoxynucleotide (CpG, a TLR9 agonist) and the Aspergillus antigen Asp f16, induced Th1 priming in dendritic cells (Bozza et al., 2002). Furthermore, adoptive transfer of Asp f16-peptide specific cytotoxic $\mathrm{T}$ lymphocytes improved survival of Aspergillus-infected mice (Sun et al., 2012). While attempts continue to develop fungal-specific vaccines, immunocompromised individuals at risk of fungal infection may have limited capacity to respond to vaccination and the solution may lie in the elucidation of more potent adjuvants to elicit some protective immunity.

Imiquimod, a TLR-7 agonist, is a topical treatment used in actinic keratosis, external genital warts, and superficial basal cell 
carcinoma. In a novel adaption of such immune augmenting therapy, Erbagci et al. (2005) reported success with the use of topical imiquimod in an immunocompetent healthy patient who had lesions on her face for over 20 years caused by Acremonium strictum. The lesions been treated unsuccessfully with topical and systemic antifungals and cryotherapy. Finally the lesions were healed after changing to topical imiquimod and oral itraconazole. de Sousa et al. (2014) also reported favorable outcomes with their use of topical imiquimod in four patients with Chromoblastomycosis, a chronic subcutaneous lesion, most commonly caused by Fonsecaea pedrosoi. Marked improvement of the lesions was reported in all the patients with or without concurrent conventional antifungal medications.

Pentraxin-3 is a soluble pattern recognition receptor and acute phase protein. Pentraxin-3 has been implicated in host innate resistance against Aspergillus infections (Garlanda et al., 2002; Bozza et al., 2014). In a cohort of patients undergoing allogenic stem cell transplantation, genetic deficiency of PTX3 leading to defective PTX3 expression was associated with increased risk of invasive aspergillosis (IA) (Cunha et al., 2014). Hypothesizing from a rat model of IA in which treatment with PTX3 reportedly improved survival and reduced lung fungal burden (Lo Giudice et al., 2010), the administration of pentraxin-3 to complement conventional therapy may be a consideration.

\section{CELLULAR THERAPY}

\section{Granulocyte Transfusion (GTX)}

Granulocyte transfusion involves transfusion of granulocytes, including neutrophils, from a donor to the patient. Neutrophils are required for killing fungal pathogens (Levitz and Diamond, 1985) and neutropenia is a well-established risk factor for IFD (Marr et al., 2002).

In theory, GTX restores the neutrophil count and augments the host's defenses against fungi. However, the actual working of GTX was fraught with many problems which made it fall out of favor. Problems included low granulocyte counts, low quality and short lifespan of the granulocytes, compounded by the advent of newer antifungal drugs - details of which have been excellently reviewed in West et al. (2017). From the 1990s, the use of recombinant cytokines such as G-CSF, and better apheresis methods, have allowed larger doses of granulocytes to be delivered to the patient and has revived interest in this method (reviewed in Ravikumar et al., 2015; West et al., 2017). Price et al. (2015) observed that doses greater than $0.6 \times 10^{9}$, ideally $1 \times 10^{9}$ granulocytes per kilogram, seemed to have beneficial outcomes. In contrast, Valentini et al. (2017) compared the variable dosages given between the RCTs and concluded that dosage of the granulocytes did not translate to any clinical benefit to the patients.

West et al. (2017) summarized 97 cases, reporting that GTX together with G-CSF yielded an overall response rate of 50$90 \%$ in IFD. The authors also described seven IFD case series using G-CSF mobilized GTX. Six reported favorable results, while the 7th (Raad et al., 2013) reported therapeutic GTX treated patients with IA had a higher risk of not responding to antifungal treatment and had higher mortality compared to controls. West et al. (2017) also described instances of bacterial and fungal infections in adult and pediatric patients receiving GTX, with a very wide range of response rates, from 0\% (Grigg et al., 1996) to $100 \%$ (Sachs et al., 2006), which makes it hard to make any conclusions.

In a Cochrane review of 11 GTX RCTs, Estcourt et al. (2015) found low grade evidence that prophylactic GTX may decrease the risk of bacterial or fungal infections. In a separate review by the same authors, they reviewed the use of therapeutic instead of prophylactic GTX (Estcourt et al., 2016). They compared 10 trials conducted from 1975 to 2015 and concluded conservatively that there is low grade evidence of GTX having any significant decrease in mortality rate. The statistical power of the 10 trials was insufficient to determine whether GTXs affected all-cause mortality. Moreover, only two trials (Seidel et al., 2008; Price et al., 2015) have been conducted within the past 15 years since G-CSF has been incorporated into routine practice to increase the granulocyte count, making the statistical power even weaker. While these two studies did not find statistical significance, both were also riddled with obstacles such as low enrolment rates and procedural obstacles, such as differences in granulocyte dosage. Disappointingly, an ongoing study of GTX, the transfusion of granulocytes for patients with FN, the GRANITE study (German Clinical Trials Register number DRKS00000218 and EudraCT number 2009-010700-28), was last updated "recruiting withdrawn before recruiting started” (Drks, 2017). RCTs of a potentially life-saving therapy are not only a scientific problem, but an ethical problem. It is very hard for clinicians and patients alike, knowing that there is a potential life-saving therapy, to withhold the therapy from the control group. It poses an ethical dilemma, on one hand, the individual patient is severely ill; on the other hand, the wider medical community needs rigorous scientific evidence.

\section{Adoptive T-Cell Therapy}

Adoptive T-cell therapy involves the harvesting of T-lymphocytes from a patient or donor's blood, stimulating the cells to grow and expand in an in vitro system, and these cells are subsequently reinfused back into the patient, primed for action.

Adoptive T-cell therapy is already in use against viral infections in HSCT patients (reviewed in Papadopoulou et al., 2016), but it is not in use yet to treat fungal infections, partly because of the technical challenges associated with the handling, manipulation and stimulation of cells. Firstly, we face technical problems of scaling up enrichment of the T-cells in accordance with good manufacturing practice (GMP) procedures for clinical use. Many groups have come up with ingenious strategies with the aim to target multiple pathogens in a shorter period of time (Tramsen et al., 2008; Khanna et al., 2011; Tramsen et al., 2013; Stuehler et al., 2015). Bacher et al. (2015) reported success on a method to prepare and isolate GMP-compliant T-helper cells reactive to Aspergillus fumigatus for adoptive transfer. The protocol and Aspergillus fumigatus lysate will be used in the ongoing clinical trial (Prüfplancode: 01-13IDWUE, EudraCT Nr. 2013-002914-11, German Clinical Trials Register number DRKS00007890) to assess their in vivo safety and efficacy. Next, 
the modality of immunosuppression also matters. For example, immunosuppressants such as cyclosporine A, commonly used in solid organ and stem cell transplantation compromises optimal T-cell response (Tramsen et al., 2014). Adoptive T-cell therapy might be most effective in transplantation settings where antiGVHD prophylaxis is not required, such as T-cell depleted HSCT (reviewed in Papadopoulou et al., 2016).

As of now, there has only been one clinical trial for adoptive T-cell therapy in the context of patients with IFD. Perruccio et al. (2005) showed that 9 out of 10 transplant patients with IA had resolution of Aspergillus infection after receiving a single dose of Aspergillus-specific T-cell clones, compared to a response rate of only $53 \%$ (7 out of 13) in the control group. All nine subjects who had a resolution of infection also had normalization of galactomannan antigenemia levels within 6 weeks, while the controls had higher levels $(P<0.002$ vs. controls). Another clinical trial (NCT02843321), reported by Sutrave et al. (2017), used a mix of donor-derived, infection specific T-cells against Aspergillus, and viruses such as AdV, influenza, EBV, CMV, VZV, and BKV. The study was completed at the end of 2017 and its results had not been published at the time of writing this paper. To date, the promise of adoptive T-cell therapy is best epitomized by the success recently reported by Tzannou et al. (2017) using third party-derived virus-specific T-cells against BKV, CMV, EBV, $\mathrm{AdV}$, and HHV-6 in HSCT patients. We eagerly await the results of more such clinical trials in the pipeline for adoptive T-cell therapy to become a reality for patients with IFD.

\section{Chimeric Antigen Receptor (CAR) T-Cell Therapy}

In this therapy, T-cells are modified with a disarmed genetically engineered virus to express a CAR. The CAR modification allows T-cells to execute their killing command without the need to bind to other receptors. In 2017, two CAR T-cell therapies were approved by the U.S. FDA for use in cancer, Kymriah (tisagenlecleucel) for B-cell acute lymphoblastic leukemia patients (FDA, 2017b) and Yescarta (axicabtagene ciloleucel) for B-cell lymphoma (FDA, 2017a). The approval of these CAR T-cell therapies was a historic action, making it the first gene therapy available in the United States.

There has only been one CAR T-cell construct adapting the fungal receptor Dectin-1 for Aspergillus to activate T-cells via chimeric CD28 and CD3- $\zeta$ (Kumaresan et al., 2014). There are concerns on the potential limitations of CAR T-cell therapy which may hamper its use in IFD. Side effects observed in patients undergoing the two approved CAR T-cell therapies included cytokine release syndrome and neurological toxicities (Neelapu et al., 2017; Schuster et al., 2017). Thus, we hope that technological progress will help surmount these difficulties to make CAR T-cell therapy for IFD a reality in the future.

\section{Natural Killer (NK) Cell Therapy}

Natural killer cell therapy, also known as adoptive transfer of NK cells, is the transfer of NK cells from a donor to a patient. The NK cells, unlike T-cells, do not need to be antigen-primed as they are activated whenever they do not receive the inhibitory self-MHC I receptor binding (reviewed in Dahlberg et al., 2015).

In vitro human NK cells were demonstrated to have activity against a wide spectrum of fungi - Aspergillus fumigatus (Park et al., 2009; Bouzani et al., 2011; Schneider et al., 2016), C. albicans (Voigt et al., 2014), Rhizopus oryzae (Schmidt et al., 2013), Paracoccidioides brasiliensis (Jimenez and Murphy, 1984), Cryptococcus neoformans (Levitz et al., 1994), and various clinical isolates of the mucormycetes (Schmidt et al., 2016).

Currently, NK cell therapy is in its trial stage for cancers. Preemptive NK cell therapy has been trialed in a phase II clinical trial in haploidentical HSCT patients to prevent graft failure and to add antitumor effects with little risk of GVHD (Stern et al., 2012). The results seem to provide no apparent benefit or serious adverse events to patients at the dosage used. In the context of IFD, there has been no clinical trial to date using NK cells to treat IFD. Thus, NK cell therapy shows potential as an immunotherapeutic option against IFD, though much more work has to be done to prove its efficacy in human trials.

\section{MONOCLONAL ANTIBODIES (mAbs)}

Therapeutic mAbs progression into clinical trials are lacking for IFD, currently most of the mAbs for fungi are developed for diagnostic purposes. At the time of writing this paper, only two therapeutic $\mathrm{mAbs}$ for fungi have made it past phase I trials, $18 \mathrm{~B} 7$ and Efungumab.

$18 \mathrm{~B} 7$ is a murine-derived antibody against the polysaccharide capsule of C. neoformans (Casadevall et al., 1998). In a phase I trial it was well tolerated in an HIV-positive subjects (Larsen et al., 2005). 18B7 mAb was subsequently modified with a radioisotope for radio-immunotherapy, which were effective in prolonging survival and reducing $C$. neoformans burden in mice, while sparing damage to epithelial or macrophage-like cells (Bryan et al., 2013). 18B7 behaved as a catalytic antibody that catalyzes hydrolysis of the C. neoformans polysaccharide capsule (Bowen et al., 2017). Other than the academic study of 18B7, the progress of $18 \mathrm{~B} 7$ to phase II/III clinical trials has been on hold due to a lack of industrial support (Ostrosky-Zeichner et al., 2010).

Mycograb, also known as Efungumab, had made it through a multinational phase II clinical trial. Its modality of action was to target cell wall-associated fungal heat shock protein 90 and to synergize the antifungal activity of amphotericin B (Matthews et al., 2003). It had been tested to work together with standard antifungal therapy and showed a good overall response rate of $84 \%$ (Mycograb) vs. $49 \%$ (controls), reducing overall Candida-related mortality from $18 \%$ (controls) to $4 \%$ (Mycograb) (Pachl et al., 2006). However, the production of Mycograb was fraught with manufacturing quality control issues, and it was refused market authorization by the European Medicines Agency (EMA, 2007). Mycograb's purported capacity to augment amphotericin B was later thought to be nonspecific (Richie et al., 2012). An alternative C28Y variant was produced, but disappointingly did not demonstrate the same in vitro or in vivo efficiency as Mycograb (Louie et al., 2011; Richie et al., 2012). Another new potential mAb 
awaiting further development is a humanized mAb, mAbP6E7, against the cutaneous mycotic pathogen Sporothrix (de Almeida et al., 2017). We hope that in due time, we will see more support and initiatives in the field of therapeutic antibody development.

\section{IMMUNOMODULATION BY OTHER SUBSTANCES}

Most pathogens induce cytokine production as part of the host immune response, which normally is a beneficial response to recruit leukocytes and eliminate the pathogen. In some situations, infections induce an excessively large inflammation, or a cytokine storm. Uncontrolled, this can sometimes cause more damage to the host than the pathogen itself, especially when the patient is suffering from other comorbidities. As such, immunomodulatory agents to attenuate the overactive immune response may benefit the patient (Chai et al., 2011).

\section{Vitamins}

Vitamin D, also known as 25-hydroxyvitamin D, is produced by our bodies upon exposure to sunlight. Beyond its effects on bone, vitamin $\mathrm{D}$ also modulates the immune system. Vitamin D generally skews the immune response toward an anti-inflammatory profile. Interestingly, Candida-infected mice receiving low doses of vitamin $\mathrm{D}$ were reported to have significantly decreased fungal burden in their kidneys, compared with either control mice which did not receive vitamin $\mathrm{D}$, or mice which received high doses of vitamin $\mathrm{D}$ (Lim et al., 2015). These observations were accounted for through low dose vitamin D-mediated suppression of SOCS3 and induction of vitamin $\mathrm{D}$ receptor binding to the vitamin D-response elements in the promoter of the gene encoding INF- $\gamma$. This highlighted the bimodal influence of vitamin D in host response against Candida and that extreme ranges of levels of vitamin D had adverse effects on the immunity, and Candida resistance. Studies are being done to determine if this dose-dependent effect of vitamin D supplementation for IFD can be translated into humans. In a phase I, open label study (NCT01222273), Nguyen et al. (2015) gave 6 months of daily vitamin D3 (cholecalciferol) supplementation to cystic fibrosis patients who had low levels of vitamin D and allergic bronchopulmonary aspergillosis. The study found Aspergillus induced IL-13 and IgE levels to be significantly decreased after 24 weeks $(P=0.04)$. Vitamin $\mathrm{D}$ supplementation as a therapy is an easily available, economical, and attractive intervention. Taking into account all these studies, it seems that cautious vitamin D supplementation may improve outcomes in fungal-related diseases.

Vitamin A, best known for its role in good eyesight, has been known to also have immunomodulatory effects. atRA is a metabolite of vitamin A, added to monocytes, challenged with UV-treated C. albicans, has been found to produce lower levels of pro-inflammatory cytokines TNF $\alpha$, IL-6, and IL-12b, when compared to monocytes without atRA (Klassert et al., 2014). Klassert et al. (2017) studied the gene expression of monocytes incubated with atRA or vitamin D, and challenged with Aspergillus fumigatus and C. albicans. They found generally both atRA and vitamin D downregulated most genes related to cytokine and chemokine activity, and also regulated noncoding RNAs involved in immunity (Riege et al., 2017). A review of 6 cystic fibrosis patients with chronic necrotizing pulmonary aspergillosis, who received voriconazole and vitamin A supplementation, reported that all the patients survived and recovered, though a higher incidence of visual disturbances were reported (Cheng et al., 2010). The use of vitamin A or atRA has not been tested in animal models of fungal infection, and more studies have to be conducted to understand the feasibility of vitamin $\mathrm{A}$ as an adjunct immune modulator against the fungi.

\section{Repurposing of Existing Drugs}

Drug development is a long, costly and arduous process, with only a handful of drug candidates eventually making it to the bedside. Repurposing of existing drugs has the benefit of shortcutting the process, while saving precious time and money. The drugs are already recognized, optimized in terms of pharmacodynamics, pharmacokinetics, safety and toxicity profiles, and have gone through the necessary regulatory processes. Some drugs that have been used for various diseases have been repurposed as adjuncts for the use in fungal infections. We would like to mention a few of these repurposed drugs that have made it to the clinical trial stage.

Deferasirox, is an iron chelator used for treatment of iron overload. In a mouse model, it was found to be efficacious against Rhizopus, which requires iron for growth (Ibrahim et al., 2007). A randomized, double-blinded, placebo-controlled trial repurposing deferasirox as an adjunct agent to amphotericin $\mathrm{B}$ therapy did not yield a positive result (DEFEAT Mucor study, NCT00419770; Spellberg et al., 2012). Unfortunately, statistical analysis showed that patients in the deferasirox arm of the trial were also more likely to have active malignancy, neutropenia and have received corticosteroid therapy, and patients given deferasirox had a higher mortality compared to the controls, which made interpretation of results inconclusive at best (Donnelly and Lahav, 2012; Spellberg et al., 2012). Optimistically, after the study results was published, Soman et al. (2012) reported seven patients who were prescribed deferasirox with standard treatment for mucormycosis where adequate surgical debridement was not feasible. The condition of these patients improved, and the authors attributed the success of the treatment to the patient group having minimal comorbidities. Thus, further RCTs are needed to establish the efficacy of this treatment.

Other drugs have had better results, such as the ASTRO-CM study (NCT01802385) which repurposed sertraline, a selective serotonin reuptake inhibitor antidepressant, as an adjunctive treatment for HIV-associated Cryptococcus meningitis (Rhein et al., 2016). Sertraline was found to have a growth-inhibitory effect, inhibiting the fungal protein synthesis (Zhai et al., 2012; Trevino-Rangel Rde et al., 2016). In vivo, sertraline reduced Cryptococcus burden in the mouse brain $(P<0.05)$, comparable to fluconazole (Zhai et al., 2012; Trevino-Rangel Rde et al., 2016). In the brain tissue, sertraline was concentrated 22 times 
higher than in the blood (Lewis et al., 2013), making it an ideal candidate for infection involving the central nervous system. Results of the trial were promising the rate of fungal clearance was better in the sertraline group, with suggestive decreased incidence of paradoxical IRIS and lower disease recurrence. The authors speculated that sertraline might also have immunoregulatory effects to explain the decreased IRIS and lower recurrence, but it requires more evidence from in vitro studies and RCTs.

Tamoxifen, an estrogen receptor antagonist, is currently in a phase II clinical trial to determine if it augments antifungal therapy against Cryptococcus meningitis (NCT03112031). Tamoxifen, like sertraline, crosses the blood-brain barrier and achieves high concentrations in the brain (Morello et al., 2003). Its role as an antifungal was identified, and observed to be synergistic with fluconazole (Wiseman et al., 1989; Dolan et al., 2009; Spitzer et al., 2011). In a murine model of Cryptococcus infection, the combination of tamoxifen and fluconazole reduced Cryptococcus brain burden significantly compared to untreated control $(P=0.001)$ (Butts et al., 2014). Tamoxifen worked by interfering with calcium homeostasis as an inhibitor of calmodulin (Beggs, 1993; Dolan et al., 2009; Butts et al., 2014). We hope to hear their results in future updates of the trial.

Other exciting drugs being considered include auranofin and ebselen, Auranofin is a gold complex originally used to treat rheumatoid arthritis. Ebelsen is an organoselenium drug with an anti-inflammatory predisposition. Both auranofin and ebelsen inhibit the thioredoxin reductase pathway (reviewed in May et al., 2018). Pivotal to this mode of action is that the thioreductase system, which is critical in maintaining cells in a reduced state, is different in humans (and hence are not affected by auranofin and ebelsen) in contrast to bacteria and fungi. Auranofin induces heme oxygenase 1 expression, which degrades heme and has anti-inflammatory properties (Kobayashi et al., 2006). Ebselen induces reactive oxygen species-mediated cytotoxicity in yeast (Azad et al., 2014). Both had been found to have in vitro activity against Aspergillus and Candida (Ngo et al., 2016; Wiederhold et al., 2017). It is to be highlighted though that the activity of auranofin and ebelsen are not specific to fungi, but also all bacteria, helminths and some protozoans (reviewed in May et al., 2018). Nonetheless should their efficacy be further validated, these drugs will have the potential to be studied in a range of antimicrobial human clinical trials.

\section{PROMISING FUTURE THERAPIES}

\section{Immunomodulation Through Probiotics and the Microbiome}

To defeat pathogenic microorganisms, it used to be thought that we had to kill them with antimicrobials. There has been a paradigm shift in recent years - the concept of defeating microorganisms with microorganisms. The successful utilization of donor fecal transfer for recurrent Clostridia infection is one such example (van Nood et al., 2013). Probiotics, the beneficial microorganisms, work by an array of mechanisms, from directly competing with the fungi for nutrients and secreting inhibitory metabolites, to modulating cytokine levels (reviewed in AbedinDo et al., 2015; Matsubara et al., 2016).

Studies with probiotics have generally yielded beneficial outcomes for patients. We focus on the probiotic yeast, Saccharomyces boulardii, which has been used to counter diarrhea. S. boulardii is able to modulate the immune system. It was found to stimulate secretory IgA production (Buts et al., 1990; Rodrigues et al., 2000; Qamar et al., 2001), and also modulates signaling pathways NF-кB and MAP kinase, ERK1/2, and p38 signaling - the details are excellently summarized in the review by Pothoulakis (2009). In a mouse model, S. boulardii was shown to work antagonistically to Candida species (Ducluzeau and Bensaada, 1982), inhibiting Candida gut translocation (Berg et al., 1993), inhibiting fungal hyphal and biofilm formation (Krasowska et al., 2009), and decreasing inflammation and Candida gut colonization (Jawhara and Poulain, 2007). A note of caution though, the administration of probiotics in neonates, severely debilitated, and immunocompromised patients is still subject to debate as there have been case reports of iatrogenic S. boulardii infections (reviewed in Goldenberg et al., 2015).

Clinical trial results are also optimistic for the use of S. boulardii as prophylaxis or adjunct therapy. A clinical trial compared the rates of fungal infections in very low birth weight infants with nystatin prophylaxis vs. oral S. boulardii; it found lower rates of Candida colonization and less feeding intolerance in those infants administered with $S$. boulardii (Demirel et al., 2013). A meta-analysis of 22 clinical trials found that pediatric patients with diarrhea had better outcomes when given $S$. boulardii probiotic supplementation (Feizizadeh et al., 2014). From the large body of evidence on the efficacy of $S$. boulardii supplementation, we believe it will be a useful adjunct therapy for fungal infection that is easily available and economically feasible for the patient. The advantages of probiotic supplementation too, are that patients see it as a "natural" alternative to drugs and may be more willing to consume it.

Our microbiota has been coined as an organ, forgotten and now rediscovered (O'Hara and Shanahan, 2006). The term microbiome is used to refer to all the microorganisms in our body, but usually refers to the bacterial aspect of the microbiome, as it is most researched. The mycobiome in particular, is termed to refer to the more neglected fungal aspect of the microbiome (reviewed in Sam et al., 2017). Both the micro- and myco-biomes play a significant role in modulating the immune system, also known as gut-immune axis. The gut microbiota can produce many metabolites that modulate the immune system, such as vitamins, short chain fatty acids, medium-chain fatty acids, secondary bile acids, bacteriocins, and antimicrobial peptides (reviewed in Sam et al., 2017).

The components of the mycobiome matter in disease outcomes. Chemically induced colitis in dectin-1 deficient mice was observed to be worse than in control mice (Iliev et al., 2012). On analysis of the dectin-1 deficient mice's mycobiome, they found Candida tropicalis, an opportunistic pathogen, constituted a large portion of the mycobiome. Furthermore, fungal invasion of the colonic wall explained the poor outcome of these dectin-1 deficient mice. Upon supplementation of the dectin-1 deficient mice with either $S$. fibuligera, a non-pathogenic fungus, or 
TABLE 1 | Summary of human clinical studies involving immunomodulatory therapies.

\begin{tabular}{llll}
\hline Reference & $\begin{array}{l}\text { Study type/clinical } \\
\text { phase }\end{array}$ & Study cohort/Patients & $\begin{array}{l}\text { Treatment outcome/therapeutic } \\
\text { response }\end{array}$
\end{tabular}

\section{Cytokine therapy}

G-CSF, GM-CSF

Clark et al. (2005)

Meta-analysis of 13 clinical trials
Total of 1,518 patients in the 13 trials
Six studies used G-CSF, six used GM-CSF, one used G-CSF, and GM-CSF treatment

$\begin{array}{ll}\text { Mhaskar et al. } & \text { Meta-analysis of } 14 \\ \text { (2014) } & \text { RCTs }\end{array}$

Wan et al. (2015)

M-CSF

Nemunaitis et al. Phase I/II trials (1993)

Interferon-gamma

Pappas et al. (2004)

Jarvis et al. (2012)

Open label RCT

Delsing et al. (2014)

Open label clinical case series

Cytokine agonist therapy

Erbagci et al. (2005) Case report

de Sousa et al. Case series

(2014)
Phase II, double-blind, placebo-controlled trial
1,553 patients with chemotherapy-induced febrile neutropenia in 14 trials

206 allo-HSCT patients

Long term follow up of 46 stem cell transplantation patients

70 HIV positive patients with acute Cryptococcus meningitis (23 controls, 25 receiving $100 \mu \mathrm{g}$ of IFN- $\gamma, 22$ receiving $200 \mu \mathrm{g}$ of IFN- $\gamma$ )

88 HIV-infected patients infected with Cryptococcus meningitis (31 controls, 29 receiving 2 doses, and 20 receiving 6 doses)
Prophylactic treatment with G-CSF, GM-CSF, or $\mathrm{G}-\mathrm{CSF}+\mathrm{GM}-\mathrm{CSF}$

The experimental group was given recombinant human M-CSF daily with conventional antifungal treatment.

Patients received 100 or $200 \mu \mathrm{g}$ of IFN $-\gamma$ or placebo three times a week for 10 weeks, in addition to standard therapy consisting of intravenous amphotericin B, with or without flucytosine, for 14 days, followed by fluconazole.

Patients were randomized to receive standard antifungal therapy for 2 weeks (amphotericin B plus flucytosine), or standard antifungal therapy plus two doses of $100 \mu \mathrm{g} \mathrm{IFN-} \gamma$, or standard antifungal therapy plus six doses of $100 \mu \mathrm{g}$ IFN $\gamma$ The experimental group was administered IFN- $\gamma, 50 \mu \mathrm{g} / \mathrm{m}^{2}$ body surface, three times a week, in addition to standard antifungal therapy placebo controls, 3 with IFN5 more treated with IFN- $\gamma$ included as therapy of last resort

One patient with recalcitrant hyalohyphomycosis caused by Acremonium strictum

Four patients with Chromoblastomycosis, caused by Fonsecaea pedrosoi
Imiquimod topical 5\% cream applied to area of fungal lesions and oral itraconazole antifungal treatment

Imiquimod topical 5\% cream applied to area of fungal lesion, with or without antifungal treatment
G-CSF and/or GM-CSF use reduced the duration of neutropenia and the length of stay during febrile neutropenia episodes; however, it was inconclusive whether administration improved infection-related mortality

Shorter duration of neutropenia, faster recovery of fever, shorter empiric antibiotic use, administration of the CSFs did not improve overall mortality and infection (including fungal)-related mortality

IFD-related mortality was lower in GM-CSF, G-CSF + GM-CSF group

There was better survival in the patients who received $\mathrm{M}-\mathrm{CSF}$, compared against historical controls ( $27 \%$ vs. $5 \%$ )

Patients who received IFN- $\gamma$ showed a trend toward rapid sterilization of cerebrospinal fluid (CSF). Lower dosage of $100 \mu \mathrm{g} \mathrm{IFN}-\gamma$ achieved similar results as the $200 \mu \mathrm{g}$ IFN- $\gamma$ recipients

The IFN $\gamma$ therapies caused significantly faster CSF Cryptococcus clearance, with no significant increase in adverse events. Two doses of IFN- $\gamma$ yielded similar outcomes as six doses

Five of eight patients treated with IFN- $\gamma$ recovered from the IFD. Two of the patients admitted into the study as last resort passed away due to infectious complications. One of eight was lost to follow up. All three patients in the control group recovered

Full regression of the fungal lesions after 2 months of therapy, and complete regression of all clinical and hematological abnormalities

After application of imiquimod cream, two patients had complete clearance of infection. The other two patients saw improvement after using imiquimod 
TABLE 1 | Continued

\begin{tabular}{llll}
\hline Reference & $\begin{array}{l}\text { Study type/clinical } \\
\text { phase }\end{array}$ & Study cohort/Patients & $\begin{array}{l}\text { Treatment outcome/therapeutic } \\
\text { response }\end{array}$
\end{tabular}

\section{Cellular therapy}

Granulocyte transfusion (GTX)

West et al. (2017)

Review of GTX from case series, prophylactic GTX in eight RCTs, therapeutic GTX in five RCTs

Estcourt et al. (2015, 2016)

Meta-analysis of 11 RCTs and 10 RCTs, respectively
Adult and pediatric patients with underlying malignancy and IFD and/or bacterial infection

653 participants in total (Estcourt et al., 2015); 587 participants in total (Estcourt et al., 2016)
The experimental groups were given prophylactic GTX or therapeutic GTX

Estcourt et al. (2015) - The experimental groups were given prophylactic GTX vs. controls without GTX

Estcourt et al. (2016) - The experimental group were given therapeutic GTX vs. controls without GTX

\section{Raad et al. (2013) \\ Case series, retrospective review}

Phase III randomized controlled trial

Phase III, open label, multicenter randomized controlled trial
Price et al. (2015), The RING study
Adoptive T-cell therapy

Perruccio et al. (2005)
Phase I/II clinical trial

Transplant patients with evidence of IA ( $n=10)$, controls $(n=13)$
Patients with hematological malignancies, prolonged neutropenia, and proven or probable invasive aspergillosis (IA)

74 patients with neutropenia were randomized to receive standard antimicrobial/antifungal therapy plus GTX ( $n=38)$ vs. the no-GTX control arm $(n=34)$

Patients with neutropenia from 14 centers were randomized to receive standard antimicrobial therapy plus GTX ( $n=56)$, or the control arm without GTX $(n=58)$

38 patients with 45 viral infections, consisting of 31 with a single virus infection and seven with two virus infections
Retrospective review of outcomes with or without therapeutic GTX

Therapeutic GTX was administered to patients, in addition to standard antimicrobial/antifungal therapy

Patients received daily GTX until neutrophil count recovery, resolution or improvement of infection, toxicity or 42 days, whichever was earlier. G-CSF was used to increase the granulocyte count in donors

T-cells from donors were stimulated with Aspergillus antigen, selected for non-recipient reactive clones, and expanded. Patients were randomized to receive treatment with liposomal amphotericin B alone, with or without, single infusion of donor pathogen-specific T-cells

Virus-specific T-cell lines for five viruses, Epstein-Barr virus (EBV), adenovirus (AdV), cytomegalovirus (CMV), BK virus (BKV), and human herpesvirus 6 (HHV-6) were manufactured from donors. All patients had at least a single infusion of partially
Low-grade evidence that prophylactic GTX may reduce the incidence of fungaemia, but non-selective prophylaxis for all neutropenic patients does not prevent mortality due to IFD Both Estcourt et al. $(2015,2016)$ found that prophylactic and therapeutic GTX, respectively, had low grade evidence for decreasing the risk of bacterial or fungal infections, or decreasing mortality rate. There was insufficient evidence to detect significant differences in mortality due to infection between GTX-treated patients and controls, and the GTX efficacy may be dose-dependent. The statistical power of the 10 trials is insufficient to determine whether GTXs affected all-cause mortality

Therapeutic GTX treated patients with IA had a higher risk of not responding to antifungal treatment and had higher mortality compared to controls

No statistical significance between the two groups

No statistical significance was found between the GTX arm and the control arm, challenges encountered included low enrolment and variation in granulocyte dosage. Subjects who received $>0.6$ billion granulocytes $/ \mathrm{kg}$ tended to have better outcomes than those receiving a lower dose of granulocytes

Nine out of 10 patients had resolution of Aspergillus infection after receiving a single dose of Aspergillus-specific T-cell clones, compared to a response rate of only $53 \%$ (7 out of 13 ) in the control group

The patients had cumulative complete or partial response rate of $92 \%$. Of special mention is by week 6,13 of 14 patients with BKV-associated hemorrhagic cystitis had complete resolution of gross hematuria 
TABLE 1 | Continued

\begin{tabular}{llll}
\hline Reference & $\begin{array}{l}\text { Study type/clinical } \\
\text { phase }\end{array}$ & Study cohort/Patients & Intervention \\
response
\end{tabular}

HLA-matched virus-specific

T-cells, 11 had a second

infusion after 4 weeks, and four

patients had three infusions

\section{Monoclonal antibodies}

Larsen et al. (2005) Phase I clinical trial
20 HIV-positive patients who had a history of culture positive Crypotcoccus meningitis, with resolution of symptoms at least 12 weeks prior to the study

139 patients who had culture-positive Candida infection
Patients were divided into six groups of escalating doses of 18B7 therapeutic monoclonal antibody against

C. neoformans. The changes in serum cryptococcal antigen titres were monitored

The experimental group received intravenous Mycograb every $12 \mathrm{~h}$ for 5 days plus lipid-amphotericin B, while controls received lipid-amphotericin B alone

\section{Immunomodulation by other substances}

Vitamins
Nguyen et al. (2015)

Phase I, open label clinical trial

Repurposing of existing drugs

Spellberg et al.

(2012), DEFEAT

Mucor study
Phase II, randomized, double-blinded, placebo-controlled trial
Cystic fibrosis patients who had low levels of vitamin $D$ and allergic bronchopulmonary aspergillosis

20 patients with proven or probable mucormycosis, 11 with deferasirox treatment, and nine placebo
The experimental group was given 6 months of daily vitamin D3 (cholecalciferol) supplementation

Patients were randomized to receive treatment with liposomal amphotericin B plus/minus deferasirox (20 mg/kg/day for 14 days)
Rhein et al. (2016), ASTRO-CM study
Open label, dose-finding study
172 HIV-infected adult patients with Cryptococcus meningitis received adjunctive sertraline at addition to standard antifungal therapy
The experimental group doses of $100-400 \mathrm{mg} /$ day, in

\section{Promising future therapies}

Immunomodulation through probiotics and the microbiome

$\begin{array}{lll}\text { Demirel et al. (2013) } & \text { Randomized, } & \text { Very low birth weight, preterm } \\ & \text { comparative trial } & \text { infants with gestational age } \\ & \leq 1,500 \mathrm{~g}\end{array}$

Pediatric patients with diarrhea

The infants were either given oral Saccharomyces boulardii prophylaxis (five billion colony forming unit per day) added to breast milk or formula once a day, starting with the first feed until discharged, or oral nystatin suspension every $8 \mathrm{~h}$

The experimental group was given $S$. boulardii
18B7 antibodies was generally well tolerated at lower doses. At higher concentrations above $1 \mathrm{mg} / \mathrm{kg}$, subjects developed infusion-associated back and muscle pain. They established that 18B7 can be safely given to subjects in doses below $1 \mathrm{mg} / \mathrm{kg}$

The complete overall response by day 10 was $84 \%$ for the Mycograb group and $48 \%$ for controls, and other indicators were also better for the Mycograb group, such as, better clinical response rate,

Candida-attributable mortality, and rate of culture-confirmed clearance of the infection

Aspergillus induced IL-13 and IgE levels to be significantly decreased after 24 weeks $(P=0.04)$

Patients given deferasirox had a higher mortality compared to the controls. The patients in the deferasirox arm of the trial were more likely to have active malignant cancer, neutropenia and corticosteroid therapy, and less likely to receive concurrent antifungals, which makes interpretation of results inconclusive

The rate of fungal clearance was better in the sertraline group, led to lower recurrence, and possibly decreased incidence of paradoxical immune reconstitution inflammatory syndrome

In the S. boulardii group, there was lower rates of sepsis, Candida colonization and less feeding intolerance. S. boulardii was not found to grow in any of the positive blood cultures. Both groups observed no serious effects from the $S$. boulardii and nystatin

S. boulardii supplementation resulted in better outcomes, such as shorter duration of diarrhea, and reduced stool frequency. It did not cause any serious adverse events 


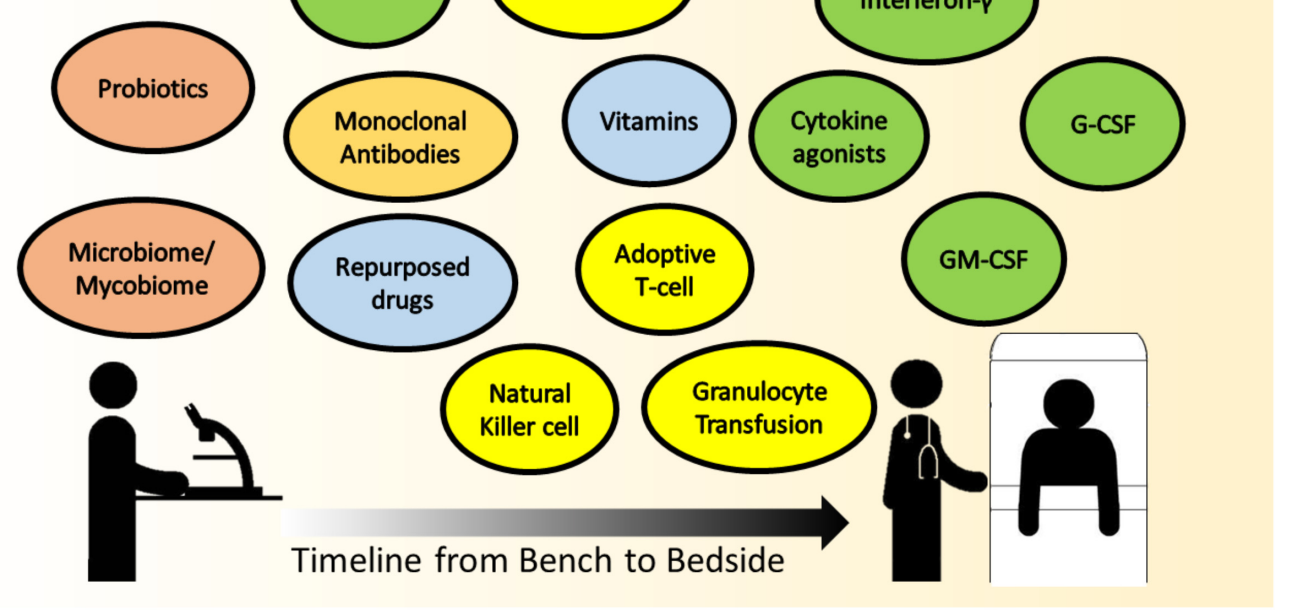

FIGURE 1 | The timeline approximating the translation of the various immune-modulating strategies for IFD, from scientific research (bench) to clinical implementation (bedside). Colored circles represent the therapy headings mentioned in this paper, green - cytokine therapy, yellow - cellular therapy, orange monoclonal antibodies, blue - other immunomodulatory agents, and peach - promising future therapies,. Therapies that are in scientific research and have the potential to be translated are on the left, with a continuum gradient of translation toward therapies that are closer to bedside use toward the right.

with C. tropicalis, mice with S. fibuligera supplementation had much less weight loss than the mice that had C. tropicalis supplementation. It was also noted that there was less production of IL-17 and IFN- $\gamma$ in S. fibuligera supplemented mice compared to $C$. tropicalis supplemented mice. Although S. fibuligera is not a probiotic, the study showed the importance of the microbial flora and how it could alter the outcome of colitis in mice.

Current therapy for patients undergoing immunosuppression frequently involves broad spectrum antibiotics and antifungal prophylaxis to reduce the risks of infection (reviewed in Maertens et al., 2012). The extent of their impact on both the microbiome and mycobiome, however, is not known - highlighting the collateral effects of broad spectrum antimicrobials on the commensal microbial flora which acts antagonistically against pathogens. More has to be done to determine the immunological impact of micro- and myco-biome interactions in humans, which will pave the way for future therapies to modulate the immune system through the micro- and mycobiota.

A summary of the clinical studies in this paper can be found in Table 1.

\section{CONCLUDING REMARKS - ARE WE CLOSER?}

With the advancements in medical sciences, there is a trend toward a combination of treatment strategies to optimize management of complicated medical conditions. As has been reviewed here, immunomodulatory options for IFD are abound, but which strategies are most likely to be translatable to the bedside in the near future? Colony stimulating factors, mainly G-CSF and to a lesser extent GM-CSF, are being administered in chemotherapy-induced FN to reduce duration of neutropenia (Smith et al., 2006). Recombinant human IFN- $\gamma$ is not a standard of care in IFD, but is selectively given with conventional antifungals in patients with difficult-to-treat IFD. The practice of GTX in IFD remains controversial. should be highlighted that indications for GTX, if so instituted, ought to be as a temporary measure to a more definitive end-point, such as the perceived recovery of neutrophil count. Patients with chronic diseases, and those requiring repeated hospitalizations are at risk of low vitamin D levels (Lim et al., 2015). Some units do actively screen and replenish vitamin $\mathrm{D}$ in their patients as per international guidelines, not specifically against IFD, but rather for bone health (Aloia, 2011). Adoptive T-cell transfer and CAR $\mathrm{T}$-cell therapies are extremely novel and have the potential to be game-changers in treatment, perhaps in the foreseeable future. Adoptive T-cell infusion is already being administered against viral infections post-stem cell transplant, such as CMV (Blyth et al., 2013; Tzannou et al., 2017). The manipulation of the microand mycobiome, as state-of-art and intellectually stimulating, remains at this point an academic venture for understanding dysbiosis and disease pathogenesis. The timeline approximating the translation of the various immune modulating strategies for IFD to clinical implementation at the bedside is depicted in Figure 1.

It is imperative to highlight at this juncture, that in facing IFD, conventional antifungal drugs remains the backbone of any treatment. The current state of immunotherapy, at this stage, augments conventional treatment as an adjunct to optimize response and outcomes. Developing novel immunomodulatory 
strategies is at the forefront of medicine today. Considering the revolution of immunotherapies in treating cancer, immunomodulatory treatment options of IFD may ride on the wave of progress in cancer immunotherapy in the coming years.

Immunotherapy and antifungal therapy are our two-pronged strategy to win against fungi. Just like a battle in an electronic game, immunotherapy is our potion to strengthen ourselves, while antifungal therapy is our potion to weaken the enemy. In an electronic battle though, when you run out of potions, the gravest consequence is your avatar faints, you lose the battle, and you revive it after the match. In real life, patients' lives are at stake in the battle against IFD. We face a constant battle against IFD, and we hope there will be a greater awareness of the need for more research and clinical trials in the exciting realm of IFD.

\section{AUTHOR CONTRIBUTIONS}

QS and LC wrote the review paper, together with WY, CS, and MC. All authors contributed to manuscript revision, read, and approved the submitted version.

\section{REFERENCES}

Abedin-Do, A., Taherian-Esfahani, Z., Ghafouri-Fard, S., Ghafouri-Fard, S., and Motevaseli, E. (2015). Immunomodulatory effects of Lactobacillus strains: emphasis on their effects on cancer cells. Immunotherapy 7, 1307-1329. doi: $10.2217 / \mathrm{imt} .15 .92$

Aloia, J. F. (2011). Clinical review: the 2011 report on dietary reference intake for vitamin D: where do we go from here? J. Clin. Endocrinol. Metab. 96, 2987-2996. doi: 10.1210/jc.2011-0090

Armstrong-James, D., Teo, I. A., Shrivastava, S., Petrou, M. A., Taube, D., Dorling, A., et al. (2010). Exogenous interferon- $\gamma$ immunotherapy for invasive fungal infections in kidney transplant patients. Am. J. Transplant. 10, 1796-1803. doi: 10.1111/j.1600-6143.2010.03094.x

Azad, G. K., Singh, V., Mandal, P., Singh, P., Golla, U., Baranwal, S., et al. (2014). Ebselen induces reactive oxygen species (ROS)-mediated cytotoxicity in Saccharomyces cerevisiae with inhibition of glutamate dehydrogenase being a target. FEBS Open Bio 4, 77-89. doi: 10.1016/j.fob.2014.01.002

Bacher, P., Jochheim-Richter, A., Mockel-Tenbrink, N., Kniemeyer, O., Wingenfeld, E., Alex, R., et al. (2015). Clinical-scale isolation of the total Aspergillus fumigatus-reactive T-helper cell repertoire for adoptive transfer. Cytotherapy 17, 1396-1405. doi: 10.1016/j.jcyt.2015.05.011

Bandera, A., Trabattoni, D., Ferrario, G., Cesari, M., Franzetti, F., Clerici, M., et al. (2008). Interferon- $\gamma$ and granulocyte-macrophage colony stimulating factor therapy in three patients with pulmonary aspergillosis. Infection 36, 368-373. doi: 10.1007/s15010-008-7378-7

Beggs, W. H. (1993). Anti-Candida activity of the anti-cancer drug tamoxifen. Res. Commun. Chem. Pathol. Pharmacol. 80, 125-128.

Berclaz, P.-Y., Zsengellér, Z., Shibata, Y., Otake, K., Strasbaugh, S., Whitsett, J. A., et al. (2002). Endocytic internalization of adenovirus, nonspecific phagocytosis, and cytoskeletal organization are coordinately regulated in alveolar macrophages by GM-CSF and PU.1. J. Immunol. 169, 6332-6342. doi: 10.4049/jimmunol.169.11.6332

Berg, R., Bernasconi, P., Fowler, D., and Gautreaux, M. (1993). Inhibition of candida albicans translocation from the gastrointestinal tract of mice by oral administration of Saccharomyces boulardii. J. Infect. Dis. 168, 1314-1318. doi: 10.1093/infdis/168.5.1314

Blyth, E., Clancy, L., Simms, R., Ma, C. K., Burgess, J., Deo, S., et al. (2013). Donor-derived CMV-specific $\mathrm{T}$ cells reduce the requirement for CMVdirected pharmacotherapy after allogeneic stem cell transplantation. Blood 121, 3745-3758. doi: 10.1182/blood-2012-08-448977

\section{FUNDING}

MC was supported by the Synthetic Biology Initiative of the National University of Singapore (DPRT/943/09/14), the Summit Research Program of the National University Health System (NUHSRO/2016/053/SRP/05), the Ministry of Defence of Singapore (MINDEF, RE2016-074), and the Defense Threat Reduction Agency (DTRA, HDTRA1-13-1-0037). LC was supported by the Clinician Scientist Award (CSA), Individual Research Grant (IRG), Bedside \& Bench (B\&B) grants, Centre Grant and the Training Fellowship Award from the National Medical Research Council (NMRC), Singapore. LC also acknowledges the Aspiration Grant \& Summit Research Program and Bench to Bedside Grant from the National University Health System as well as the Synthetic Biology Research \& Development Program of the National Research Foundation, Singapore.

\section{ACKNOWLEDGMENTS}

We thank Dr. Sophie Dennis for proofreading the manuscript.

Bouzani, M., Ok, M., Mccormick, A., Ebel, F., Kurzai, O., Morton, C. O., et al. (2011). Human NK cells display important antifungal activity against Aspergillus fumigatus, which is directly mediated by IFN- $\gamma$ release. J. Immunol. 187, 1369-1376. doi: 10.4049/jimmunol.1003593

Bowen, A., Wear, M. P., Cordero, R. J. B., Oscarson, S., and Casadevall, A. (2017). A monoclonal antibody to Cryptococcus neoformans glucuronoxylomannan manifests hydrolytic activity for both peptides and polysaccharides. J. Biol. Chem. 292, 417-434. doi: 10.1074/jbc.M116.767582

Bozza, S., Campo, S., Arseni, B., Inforzato, A., Ragnar, L., Bottazzi, B., et al. (2014). PTX3 binds MD-2 and promotes TRIF-dependent immune protection in Aspergillosis. J. Immunol. 193, 2340-2348. doi: 10.4049/jimmunol.1400814

Bozza, S., Clavaud, C., Giovannini, G., Fontaine, T., Beauvais, A., Sarfati, J., et al. (2009). Immune sensing of Aspergillus fumigatus proteins, glycolipids, and polysaccharides and the impact on th immunity and vaccination. J. Immunol. 183, 2407-2414. doi: 10.4049/jimmunol.0900961

Bozza, S., Gaziano, R., Lipford, G. B., Montagnoli, C., Bacci, A., Di Francesco, P., et al. (2002). Vaccination of mice against invasive aspergillosis with recombinant Aspergillus proteins and $\mathrm{CpG}$ oligodeoxynucleotides as adjuvants. Microbes Infect. 4, 1281-1290. doi: 10.1016/S1286-4579(02)00007-2

Brown, G. D., Denning, D. W., Gow, N. A., Levitz, S. M., Netea, M. G., and White, T. C. (2012). Hidden killers: human fungal infections. Sci. Transl. Med. 4:165rv113. doi: 10.1126/scitranslmed.3004404

Bryan, R. A., Jiang, Z., Morgenstern, A., Bruchertseifer, F., Casadevall, A., and Dadachova, E. (2013). Radioimmunotherapy of Cryptococcus neoformans spares bystander mammalian cells. Fut. Microbiol. 8, 1081-1089. doi: 10.2217/ fmb.13.79

Buddingh, E. P., Leentjens, J., Van Der Lugt, J., Dik, W. A., Gresnigt, M. S., Netea, M. G., et al. (2015). Interferon-gamma immunotherapy in a patient with refractory disseminated candidiasis. Pediatr. Infect. Dis. J. 34, 1391-1394. doi: 10.1097/INF.0000000000000909

Buts, J.-P., Bernasconi, P., Vaerman, J.-P., and Dive, C. (1990). Stimulation of secretory IgA and secretory component of immunoglobulins in small intestine of rats treated with Saccharomyces boulardii. Dig. Dis. Sci. 35, 251-256. doi: 10.1007/BF01536771

Butts, A., Koselny, K., Chabrier-Roselló, Y., Semighini, C. P., Brown, J. C. S., Wang, X., et al. (2014). Estrogen receptor antagonists are anti-cryptococcal agents that directly bind ef hand proteins and synergize with fluconazole in vivo. mBio 5:e765-13. doi: 10.1128/mBio.00765-13

Casadevall, A., Cleare, W., Feldmesser, M., Glatman-Freedman, A., Goldman, D. L., Kozel, T. R., et al. (1998). Characterization of a murine monoclonal 
antibody to Cryptococcus neoformans polysaccharide that is a candidate for human therapeutic studies. Antimicrob. Agents Chemother. 42, 1437-1446.

Chai, L. Y., Vonk, A. G., Kullberg, B.-J., and Netea, M. G. (2011). Immune response to Aspergillus fumigatus in compromised hosts: from bedside to bench. Fut. Microbiol. 6, 73-83. doi: 10.2217/fmb.10.158

Chen, T. K., Groncy, P. K., Javahery, R., Chai, R. Y., Nagpala, P., Finkelman, M., et al. (2017). Successful treatment of Aspergillus ventriculitis through voriconazole adaptive pharmacotherapy, immunomodulation, and therapeutic monitoring of cerebrospinal fluid (1rightarrow3)- $\beta$-D-glucan. Med. Mycol. 55, 109-117. doi: 10.1093/mmy/myw118

Cheng, M. P., Paquette, K., Lands, L. C., Ovetchkine, P., Théoret, Y., and Quach, C. (2010). Voriconazole inhibition of vitamin A metabolism: are adverse events increased in cystic fibrosis patients? Pediatr. Pulmonol. 45, 661-666. doi: 10. 1002/ppul.21234

Clark, O. A., Lyman, G. H., Castro, A. A., Clark, L. G., and Djulbegovic, B. (2005). Colony-stimulating factors for chemotherapy-induced febrile neutropenia: a meta-analysis of randomized controlled trials. J. Clin. Oncol. 23, 4198-4214. doi: 10.1200/JCO.2005.05.645

Cunha, C., Aversa, F., Lacerda, J. F., Busca, A., Kurzai, O., Grube, M., et al. (2014). Genetic PTX3 deficiency and aspergillosis in stem-cell transplantation. N. Engl. J. Med. 370, 421-432. doi: 10.1056/NEJMoa1211161

Dahlberg, C. I. M., Sarhan, D., Chrobok, M., Duru, A. D., and Alici, E. (2015). Natural killer cell-based therapies targeting cancer: possible strategies to gain and sustain anti-tumor activity. Front. Immunol. 6:605. doi: 10.3389/fimmu. 2015.00605

de Almeida, J. R. F., Santiago, K. L., Kaihami, G. H., Maranhão, A. Q., De Macedo Brígido, M., and De Almeida, S. R. (2017). The efficacy of humanized antibody against the Sporothrix antigen, gp70, in promoting phagocytosis and reducing disease burden. Front. Microbiol. 8:345. doi: 10.3389/fmicb.2017.00345

de Sousa, M. G. T., Belda, J. W., Spina, R., Lota, P. R., Valente, N. S., Brown, G. D., et al. (2014). Topical application of imiquimod as a treatment for chromoblastomycosis. Clin. Infect. Dis. 58, 1734-1737. doi: 10.1093/cid/ ciu168

Delsing, C. E., Gresnigt, M. S., Leentjens, J., Preijers, F., Frager, F. A., Kox, M., et al. (2014). Interferon-gamma as adjunctive immunotherapy for invasive fungal infections: a case series. BMC Infect. Dis. 14:166. doi: 10.1186/1471-233414-166

Demirel, G., Celik, I. H., Erdeve, O., Saygan, S., Dilmen, U., and Canpolat, F. E. (2013). Prophylactic Saccharomyces boulardii versus nystatin for the prevention of fungal colonization and invasive fungal infection in premature infants. Eur. J. Pediatr. 172, 1321-1326. doi: 10.1007/s00431-013-2041-4

Dolan, K., Montgomery, S., Buchheit, B., Didone, L., Wellington, M., and Krysan, D. J. (2009). Antifungal activity of tamoxifen: in vitro and in vivo activities and mechanistic characterization. Antimicrob. Agents Chemother. 53, 3337-3346. doi: 10.1128/AAC.01564-08

Donnelly, J. P., and Lahav, M. (2012). Deferasirox as adjunctive therapy for mucormycosis. J. Antimicrob. Chemother. 67, 519-520. doi: 10.1093/jac/ dkr540

Drgona, L., Khachatryan, A., Stephens, J., Charbonneau, C., Kantecki, M., Haider, S., et al. (2014). Clinical and economic burden of invasive fungal diseases in Europe: focus on pre-emptive and empirical treatment of Aspergillus and Candida species. Eur. J. Clin. Microbiol. Infect. Dis. 33, 7-21. doi: 10.1007/ s10096-013-1944-3

Drks, D. R. (2017). Tansfusion of Granulocytes for Patients with Febrile Neutropenia (GRANITE). Deutsches Register Klinischer Studien. Available at: http://www.drks.de/drks_web/navigate.do?navigationId=trial.HTML\& TRIAL_ID=DRKS00000218 [accessed Dec 26, 2017].

Ducluzeau, R., and Bensaada, M. (1982). Comparative effect of a single or continuous administration of "Saccharomyces boulardii" on the establishment of various strains of "candida" in the digestive tract of gnotobiotic mice. Ann. Microbiol. 133, 491-501.

EMA (2007). Refusal CHMP Assessment Report for Mycograb. Londoned EMAgencyEMEACHMP2731272007edEuropeanMedicinesAgencywebsite: European Medicines Agency.

Erbagci, Z., Tuncel, A. A., Erkilic, S., and Zer, Y. (2005). Successful treatment of antifungal- and cryotherapy-resistant subcutaneous hyalohyphomycosis in an immunocompetent case with topical 5\% imiquimod cream. Mycopathologia 159, 521-526. doi: 10.1007/s11046-005-5260-z
Estcourt, L. J., Stanworth, S. J., Doree, C., Blanco, P., Hopewell, S., Trivella, M., et al. (2015). Granulocyte transfusions for preventing infections in people with neutropenia or neutrophil dysfunction. Cochrane Database Syst. Rev. 6:CD005341. doi: 10.1002/14651858.CD005341.pub3

Estcourt, L. J., Stanworth, S. J., Hopewell, S., Doree, C., Trivella, M., and Massey, E. (2016). Granulocyte transfusions for treating infections in people with neutropenia or neutrophil dysfunction. Cochrane Database Syst. Rev. 4:CD005339. doi: 10.1002/14651858.CD005339.pub2

Estrada, C., Desai, A. G., Chirch, L. M., Suh, H., Seidman, R., Darras, F., et al. (2012). Invasive aspergillosis in a renal transplant recipient successfully treated with interferon-gamma. Case Rep. Transplant. 2012:493758. doi: 10.1155/2012/ 493758

FDA (2000). FDA Approved Drug Products. Available at: (https://www.access data.fda.gov/scripts/cder/daf/index.cfm?event=overview.process\&ApplNo=10 3836:FDA,U.S

FDA (2017a). FDA Approves CAR-T Cell Therapy to Treat Adults with Certain Types of Large B-cell Lymphoma. Available: https://www.fda.gov/NewsEvents/ Newsroom/PressAnnouncements/ucm581216.htm [accessed Jan 8, 2018].

FDA (2017b). FDA Approves Tisagenlecleucel for B-cell ALL and Tocilizumab for Cytokine Release Syndrome. U.S. Food \& Drug Administration. Available: https: //www.fda.gov/drugs/informationondrugs/approveddrugs/ucm574154.htm [accessed Jan 12, 2018].

Feizizadeh, S., Salehi-Abargouei, A., and Akbari, V. (2014). Efficacy and safety of Saccharomyces boulardii for acute diarrhea. Pediatrics 134:e176-91. doi: 10. 1542/peds.2013-3950

Garlanda, C., Hirsch, E., Bozza, S., Salustri, A., De Acetis, M., Nota, R., et al. (2002). Non-redundant role of the long pentraxin PTX3 in anti-fungal innate immune response. Nature 420, 182-186. doi: 10.1038/nature01195

Goldenberg, J. Z., Lytvyn, L., Steurich, J., Parkin, P., Mahant, S., and Johnston, B. C. (2015). Probiotics for the prevention of pediatric antibiotic-associated diarrhea. Cochrane Database Syst. Rev. 12:CD004827. doi: 10.1002/14651858.CD004827. pub4

Goldman, C., Akiyama, M. J., Torres, J., Louie, E., and Meehan, S. A. (2016). Scedosporium apiospermum infections and the role of combination antifungal therapy and GM-CSF: a case report and review of the literature. Med. Mycol. Case Rep. 11, 40-43. doi: 10.1016/j.mmcr.2016.04.005

Gow, N. A. R., Van De Veerdonk, F. L., Brown, A. J. P., and Netea, M. G. (2011). Candida albicans morphogenesis and host defence: discriminating invasion from colonization. Nat. Rev. Microbiol. 10, 112-122. doi: 10.1038/nrmi cro2711

Grigg, A., Vecchi, L., Bardy, P., and Szer, J. (1996). G-CSF stimulated donor granulocyte collections for prophylaxis and therapy of neutropenic sepsis. Aust. N. Z. J. Med. 26, 813-818. doi: 10.1111/j.1445-5994.1996.tb00630.x

Hamilton, J. A. (2008). Colony-stimulating factors in inflammation and autoimmunity. Nat. Rev. Immunol. 8:533. doi: 10.1038/nri2356

Hovi, L., Saarinen-Pihkala, U. M., Vettenranta, K., and Saxen, H. (2000). Invasive fungal infections in pediatric bone marrow transplant recipients: single center experience of 10 years. Bone Marrow Transplant. 26, 999-1004. doi: 10.1038/sj. bmt. 1702654

Hume, D. A., and MacDonald, K. P. A. (2012). Therapeutic applications of macrophage colony-stimulating factor-1 (CSF-1) and antagonists of CSF-1 receptor (CSF-1R) signaling. Blood 119, 1810-1820. doi: 10.1182/blood-201109-379214

Ibrahim, A. S., Gebermariam, T., Fu, Y., Lin, L., Husseiny, M. I., French, S. W., et al. (2007). The iron chelator deferasirox protects mice from mucormycosis through iron starvation. J. Clin. Invest. 117, 2649-2657. doi: 10.1172/JCI32338

Iliev, I. D., Funari, V. A., Taylor, K. D., Nguyen, Q., Reyes, C. N., Strom, S. P., et al. (2012). Interactions between commensal fungi and the C-Type lectin receptor Dectin-1 influence colitis. Science 336, 1314-1317. doi: 10.1126/ science. 1221789

Jarvis, J. N., Meintjes, G., Rebe, K., Williams, G. N., Bicanic, T., Williams, A., et al. (2012). Adjunctive Interferon- $\gamma$ immunotherapy for the treatment of HIVassociated cryptococcal meningitis: a randomized controlled trial. AIDS 26, 1105-1113. doi: 10.1097/QAD.0b013e3283536a93

Jawhara, S., and Poulain, D. (2007). Saccharomyces boulardii decreases inflammation and intestinal colonization by Candida albicans in a mouse model of chemically-induced colitis. Med. Mycol. 45, 691-700. doi: $10.1080 / 13693780701523013$ 
Jimenez, B. E., and Murphy, J. W. (1984). In vitro effects of natural killer cells against Paracoccidioides brasiliensis yeast phase. Infect. Immun. 46, 552-558.

Kandalla, P. K., Sarrazin, S., Molawi, K., Berruyer, C., Redelberger, D., Favel, A., et al. (2016). M-CSF improves protection against bacterial and fungal infections after hematopoietic stem/progenitor cell transplantation. J. Exp. Med. 213, 2269-2279. doi: 10.1084/jem.20151975

Kelleher, P., Goodsall, A., Mulgirigama, A., Kunst, H., Henderson, D. C., Wilson, R., et al. (2006). Interferon- $\gamma$ therapy in two patients with progressive chronic pulmonary aspergillosis. Eur. Respir. J. 27, 1307-1310. doi: 10.1183/ 09031936.06.00021705

Khanna, N., Stuehler, C., Conrad, B., Lurati, S., Krappmann, S., Einsele, H., et al. (2011). Generation of a multipathogen-specific T-cell product for adoptive immunotherapy based on activation-dependent expression of CD154. Blood 118, 1121-1131. doi: 10.1182/blood-2010-12-322610

Klassert, T. E., Bräuer, J., Hölzer, M., Stock, M., Riege, K., Zubiría-Barrera, C., et al. (2017). Differential effects of vitamins A and D on the transcriptional landscape of human monocytes during infection. Sci. Rep. 7:40599. doi: 10.1038/srep40599

Klassert, T. E., Hanisch, A., Bräuer, J., Klaile, E., Heyl, K. A., Mansour, M. K., et al. (2014). Modulatory role of vitamin A on the Candida albicans-induced immune response in human monocytes. Med. Microbiol. Immunol. 203, 415-424. doi: 10.1007/s00430-014-0351-4

Kobayashi, H., Takeno, M., Saito, T., Takeda, Y., Kirino, Y., Noyori, K., et al. (2006). Regulatory role of heme oxygenase 1 in inflammation of rheumatoid arthritis. Arthritis Rheum. 54, 1132-1142. doi: 10.1002/art.21754

Krasowska, A., Murzyn, A., Dyjankiewicz, A., Łukaszewicz, M., and Dziadkowiec, D. (2009). The antagonistic effect of Saccharomyces boulardii on Candida albicans filamentation, adhesion and biofilm formation. FEMS Yeast Res. 9, 1312-1321. doi: 10.1111/j.1567-1364.2009.00559.x

Kumaresan, P. R., Manuri, P. R., Albert, N. D., Maiti, S., Singh, H., Mi, T., et al. (2014). Bioengineering $\mathrm{T}$ cells to target carbohydrate to treat opportunistic fungal infection. Proc. Natl. Acad. Sci. U.S.A. 111, 10660-10665. doi: 10.1073/ pnas. 1312789111

Larsen, R. A., Pappas, P. G., Perfect, J., Aberg, J. A., Casadevall, A., Cloud, G. A., et al. (2005). Phase I evaluation of the safety and pharmacokinetics of murinederived anticryptococcal antibody $18 \mathrm{~B} 7$ in subjects with treated cryptococcal meningitis. Antimicrob. Agents Chemother. 49, 952-958. doi: 10.1128/AAC.49. 3.952-958.2005

LeVine, A. M., Reed, J. A., Kurak, K. E., Cianciolo, E., and Whitsett, J. A. (1999). GM-CSF-deficient mice are susceptible to pulmonary group B streptococcal infection. J. Clin. Invest. 103, 563-569. doi: 10.1172/JCI5212

Levitz, S. M., and Diamond, R. D. (1985). Mechanisms of resistance of Aspergillus fumigatus conidia to killing by neutrophils in vitro. J. Infect. Dis. 152, 33-42. doi: 10.1093/infdis/152.1.33

Levitz, S. M., Dupont, M. P., and Smail, E. H. (1994). Direct activity of human $\mathrm{T}$ lymphocytes and natural killer cells against Cryptococcus neoformans. Infect. Immun. 62, 194-202.

Lewis, R. J., Angier, M. K., Williamson, K. S., and Johnson, R. D. (2013). Analysis of sertraline in postmortem fluids and tissues in 11 aviation accident victims. J. Anal. Toxicol. 37, 208-216. doi: 10.1093/jat/bkt014

Lim, J. H. J., Ravikumar, S., Wang, Y.-M., Thamboo, T. P., Ong, L., Chen, J., et al. (2015). Bimodal influence of Vitamin D in host response to systemic candida infection-vitamin D dose matters. J. Infect. Dis. 212, 635-644. doi: 10.1093/infdis/jiv033

Lo Giudice, P., Campo, S., Verdoliva, A., Rivieccio, V., Borsini, F., De Santis, R., et al. (2010). Efficacy of PTX3 in a rat model of invasive aspergillosis. Antimicrob. Agents Chemother. 54, 4513-4515. doi: 10.1128/AAC.00674-10

Louie, A., Stein, D. S., Zack, J. Z., Liu, W., Conde, H., Fregeau, C., et al. (2011). Dose range evaluation of mycograb $\mathrm{C} 28 \mathrm{Y}$ variant, a human recombinant antibody fragment to heat shock protein 90 , in combination with amphotericin B-desoxycholate for treatment of murine systemic candidiasis. Antimicrob. Agents Chemother. 55, 3295-3304. doi: 10.1128/AAC.01324-10

Maertens, J. A., Nucci, M., and Donnelly, J. P. (2012). The role of antifungal treatment in hematology. Haematologica 97, 325-327. doi: 10.3324/haematol. 2012.061952

Marr, K. A., Carter, R. A., Boeckh, M., Martin, P., and Corey, L. (2002). Invasive aspergillosis in allogeneic stem cell transplant recipients: changes in epidemiology and risk factors. Blood 100, 4358-4366. doi: 10.1182/blood-200205-1496
Matsubara, V. H., Bandara, H. M. H. N., Mayer, M. P. A., and Samaranayake, L. P. (2016). Probiotics as antifungals in mucosal candidiasis. Clin. Infect. Dis. 62 1143-1153. doi: 10.1093/cid/ciw038

Matthews, R. C., Rigg, G., Hodgetts, S., Carter, T., Chapman, C., Gregory, C., et al. (2003). Preclinical assessment of the efficacy of mycograb, a human recombinant antibody against fungal HSP90. Antimicrob. Agents Chemother. 47, 2208-2216. doi: 10.1128/AAC.47.7.2208-2216.2003

May, H. C., Yu, J.-J., Guentzel, M. N., Chambers, J. P., Cap, A. P., and Arulanandam, B. P. (2018). Repurposing Auranofin, Ebselen, and PX-12 as antimicrobial agents targeting the thioredoxin system. Front. Microbiol. 9:336. doi: $10.3389 /$ fmicb. 2018.00336

Medina-Echeverz, J., Aranda, F., and Berraondo, P. (2014). Myeloid-derived cells are key targets of tumor immunotherapy. Oncoimmunology 3:e28398. doi: 10. 4161/onci.28398

Mezidi, M., Belafia, F., Nougaret, S., Pageaux, G. P., Conseil, M., Panaro, F., et al. (2014). Interferon gamma in association with immunosuppressive drugs withdrawal and antifungal combination as a rescue therapy for cerebral invasive Aspergillosis in a liver transplant recipient. Minerva Anestesiol. 80, 1359-1360.

Mhaskar, R., Clark, O. A., Lyman, G., Engel Ayer Botrel, T., Morganti Paladini, L., and Djulbegovic, B. (2014). Colony-stimulating factors for chemotherapyinduced febrile neutropenia. Cochrane Database Syst. Rev. 10:Cd003039. doi: 10.1002/14651858.CD003039.pub2

Morello, K. C., Wurz, G. T., and Degregorio, M. W. (2003). Pharmacokinetics of selective estrogen receptor modulators. Clin. Pharmacokinet. 42, 361-372. doi: 10.2165/00003088-200342040-00004

Neelapu, S. S., Locke, F. L., Bartlett, N. L., Lekakis, L. J., Miklos, D. B., Jacobson, C. A., et al. (2017). Axicabtagene ciloleucel CAR T-cell therapy in refractory large B-cell lymphoma. N. Engl. J. Med. 377, 2531-2544. doi: 10.1056/ NEJMoa1707447

Nemunaitis, J., Shannon-Dorcy, K., Appelbaum, F., Meyers, J., Owens, A., Day, R., et al. (1993). Long-term follow-up of patients with invasive fungal disease who received adjunctive therapy with recombinant human macrophage colonystimulating factor. Blood 82, 1422-1427.

Neofytos, D., Horn, D., Anaissie, E., Steinbach, W., Olyaei, A., Fishman, J., et al. (2009). Epidemiology and outcome of invasive fungal infection in adult hematopoietic stem cell transplant recipients: analysis of multicenter prospective antifungal therapy (PATH) alliance registry. Clin. Infect. Dis. 48, 265-273. doi: $10.1086 / 595846$

Ngo, H. X., Shrestha, S. K., and Garneau-Tsodikova, S. (2016). Identification of ebsulfur analogues with broad-spectrum antifungal activity. ChemMedChem 11, 1507-1516. doi: 10.1002/cmdc.201600236

Nguyen, N. L. H., Pilewski, J. M., Celedón, J. C., Mandalapu, S., Blanchard, M. L., Dericco, A., et al. (2015). Vitamin D supplementation decreases Aspergillus fumigatus specific Th2 responses in CF patients with aspergillus sensitization: a phase one open-label study. Asthma Res. Pract. 1:3. doi: 10.1186/s40733-0150003-5

O'Hara, A. M., and Shanahan, F. (2006). The gut flora as a forgotten organ. EMBO Rep. 7, 688-693. doi: 10.1038/sj.embor.7400731

Ostrosky-Zeichner, L., Casadevall, A., Galgiani, J. N., Odds, F. C., and Rex, J. H. (2010). An insight into the antifungal pipeline: selected new molecules and beyond. Nat. Rev. Drug Discov. 9, 719-727. doi: 10.1038/nrd3074

Pachl, J., Svoboda, P., Jacobs, F., Vandewoude, K., Van Der Hoven, B., Spronk, P., et al. (2006). A randomized, blinded, multicenter trial of lipid-associated amphotericin B alone versus in combination with an antibody-based inhibitor of heat shock protein 90 in patients with invasive candidiasis. Clin. Infect. Dis. 42, 1404-1413. doi: 10.1086/503428

Paine, R., Preston, A. M., Wilcoxen, S., Jin, H., Siu, B. B., Morris, S. B., et al. (2000). Granulocyte-Macrophage colony-stimulating factor in the innate immune response to Pneumocystis carinii pneumonia in mice. J. Immunol. 164, 2602-2609. doi: 10.4049/jimmunol.164.5.2602

Papadopoulou, A., Kaloyannidis, P., Yannaki, E., and Cruz, C. R. (2016). Adoptive transfer of aspergillus-specific $\mathrm{T}$ cells as a novel anti-fungal therapy for hematopoietic stem cell transplant recipients: progress and challenges. Crit. Rev. Oncol. Hematol. 98, 62-72. doi: 10.1016/j.critrevonc.2015.10.005

Pappas, P. G., Bustamante, B., Ticona, E., Hamill, R. J., Johnson, P. C., Reboli, A., et al. (2004). Recombinant Interferon- $\gamma \mathrm{lb}$ as adjunctive therapy for AIDSrelated acute cryptococcal meningitis. J. Infect. Dis. 189, 2185-2191. doi: 10. $1086 / 420829$ 
Park, S. J., Hughes, M. A., Burdick, M., Strieter, R. M., and Mehrad, B. (2009). Early NK cell-derived IFN-\{gamma\} is essential to host defense in neutropenic invasive aspergillosis. J. Immunol. 182, 4306-4312. doi: 10.4049/jimmunol. 0803462

Perruccio, K., Tosti, A., Burchielli, E., Topini, F., Ruggeri, L., Carotti, A., et al. (2005). Transferring functional immune responses to pathogens after haploidentical hematopoietic transplantation. Blood 106, 4397-4406. doi: 10. 1182/blood-2005-05-1775

Pothoulakis, C. (2009). Review article: anti-inflammatory mechanisms of action of Saccharomyces boulardii. Aliment. Pharmacol. Ther. 30, 826-833. doi: 10.1111/ j.1365-2036.2009.04102.x

Price, T. H., Boeckh, M., Harrison, R. W., Mccullough, J., Ness, P. M., Strauss, R. G., et al. (2015). Efficacy of transfusion with granulocytes from G-CSF/dexamethasone-treated donors in neutropenic patients with infection. Blood 126, 2153-2161. doi: 10.1182/blood-2015-05-645986

Qamar, A., Aboudola, S., Warny, M., Michetti, P., Pothoulakis, C., Lamont, J. T., et al. (2001). Saccharomyces boulardii stimulates intestinal immunoglobulin A immune response to clostridium difficile toxin A in mice. Infect. Immun. 69, 2762-2765. doi: 10.1128/IAI.69.4.2762-2765.2001

Raad, I. I., Chaftari, A. M., Al Shuaibi, M. M., Jiang, Y., Shomali, W., Cortes, J. E., et al. (2013). Granulocyte transfusions in hematologic malignancy patients with invasive pulmonary aspergillosis: outcomes and complications. Ann. Oncol. 24, 1873-1879. doi: 10.1093/annonc/mdt110

Rajasingham, R., Smith, R. M., Park, B. J., Jarvis, J. N., Govender, N. P., Chiller, T. M., et al. (2017). Global burden of disease of HIV-associated cryptococcal meningitis: an updated analysis. Lancet Infect. Dis. 17, 873-881. doi: 10.1016/ S1473-3099(17)30243-8

Ravikumar, S., Win, M. S., and Chai, L. Y. A. (2015). Optimizing outcomes in immunocompromised hosts: understanding the role of immunotherapy in invasive fungal diseases. Front. Microbiol. 6:1322. doi: 10.3389/fmicb.2015. 01322

Rhein, J., Morawski, B. M., Hullsiek, K. H., Nabeta, H. W., Kiggundu, R., Tugume, L., et al. (2016). Efficacy of adjunctive sertraline for the treatment of HIV-associated cryptococcal meningitis: an open-label dose-ranging study. Lancet Infect. Dis. 16, 809-818. doi: 10.1016/S1473-3099(16)00074-8

Richie, D. L., Ghannoum, M. A., Isham, N., Thompson, K. V., and Ryder, N. S. (2012). The non-specific effect of mycograb on amphotericin B minimum inhibitory concentration. Antimicrob. Agents Chemother. 56, 3963-3964. doi: 10.1128/AAC.00435-12

Riege, K., Hölzer, M., Klassert, T. E., Barth, E., Bräuer, J., Collatz, M., et al. (2017). Massive effect on LncRNAs in human monocytes during fungal and bacterial infections and in response to vitamins A and D. Sci. Rep. 7:40598. doi: $10.1038 /$ srep40598

Rodrigues, A. C. P., Cara, D. C., Fretez, S. H. G. G., Cunha, F. Q., Vieira, E. C., Nicoli, J. R., et al. (2000). Saccharomyces boulardii stimulates sIgA production and the phagocytic system of gnotobiotic mice. J. Appl. Microbiol. 89, 404-414. doi: 10.1046/j.1365-2672.2000.01128.x

Romani, L. (2011). Immunity to fungal infections. Nat. Rev. Immunol. 11, 275-288. doi: $10.1038 /$ nri2939

Sachs, U. J. H., Reiter, A., Walter, T., Bein, G., and Woessmann, W. (2006). Safety and efficacy of therapeutic early onset granulocyte transfusions in pediatric patients with neutropenia and severe infections. Transfusion 46, 1909-1914. doi: 10.1111/j.1537-2995.2006.00996.x

Sam, Q., Chang, M., and Chai, L. (2017). The fungal mycobiome and its interaction with gut bacteria in the host. Int. J. Mol. Sci. 18:330. doi: 10.3390/ijms18020330

Schmidt, S., Schneider, A., Demir, A., Lass-Florl, C., and Lehrnbecher, T. (2016). Natural killer cell-mediated damage of clinical isolates of mucormycetes. Mycoses 59, 34-38. doi: 10.1111/myc. 12431

Schmidt, S., Tramsen, L., Perkhofer, S., Lass-Flörl, C., Hanisch, M., Röger, F., et al. (2013). Rhizopus oryzae hyphae are damaged by human natural killer (NK) cells, but suppress NK cell mediated immunity. Immunobiology 218, 939-944. doi: 10.1016/j.imbio.2012.10.013

Schneider, A., Blatzer, M., Posch, W., Schubert, R., Lass-Flörl, C., Schmidt, S., et al. (2016). Aspergillus fumigatus responds to natural killer (NK) cells with upregulation of stress related genes and inhibits the immunoregulatory function of NK cells. Oncotarget 7, 71062-71071. doi: 10.18632/oncotarget.12616

Schuster, S., Bishop, M. R., Tam, C., Waller, E. K., Borchmann, P., Mcguirk, J., et al. (2017). Global pivotal phase 2 trial of the CD19-targeted therapy CTL019 in adult patients with relapsed or refractory $(\mathrm{R} / \mathrm{R})$ diffuse large B-cell lymphoma (DLBCL) - an interim analysis. Clin. Lymphoma Myeloma Leuk. 17, S373-S374. doi: 10.1016/j.clml.2017.07.213

Scriven, J. E., Tenforde, M. W., Levitz, S. M., and Jarvis, J. N. (2017). Modulating host immune responses to fight invasive fungal infections. Curr. Opin. Microbiol. 40, 95-103. doi: 10.1016/j.mib.2017.10.018

Seidel, M. G., Peters, C., Wacker, A., Northoff, H., Moog, R., Boehme, A., et al. (2008). Randomized phase III study of granulocyte transfusions in neutropenic patients. Bone Marrow Transplant. 42, 679-684. doi: 10.1038/bmt.2008.237

Shiomi, A., and Usui, T. (2015). Pivotal roles of GM-CSF in autoimmunity and inflammation. Mediators Inflamm. 2015:568543. doi: 10.1155/2015/ 568543

Smith, T. J., Khatcheressian, J., Lyman, G. H., Ozer, H., Armitage, J. O., Balducci, L., et al. (2006). 2006 update of recommendations for the use of white blood cell growth factors: an evidence-based clinical practice guideline. J. Clin. Oncol. 24, 3187-3205. doi: 10.1200/JCO.2006.06.4451

Soman, R., Gupta, N., Shetty, A., and Rodrigues, C. (2012). Deferasirox in mucormycosis: hopefully, not defeated. J. Antimicrob. Chemother. 67, 783-784. doi: 10.1093/jac/dkr529

Spellberg, B., Ibrahim, A. S., Chin-Hong, P. V., Kontoyiannis, D. P., Morris, M. I., Perfect, J. R., et al. (2012). The Deferasirox-AmBisome therapy for mucormycosis (DEFEAT Mucor) study: a randomized, double-blinded, placebo-controlled trial. J. Antimicrob. Chemother. 67, 715-722. doi: 10.1093/ $\mathrm{jac} / \mathrm{dkr} 375$

Spitzer, M., Griffiths, E., Blakely, K. M., Wildenhain, J., Ejim, L., Rossi, L., et al. (2011). Cross-species discovery of syncretic drug combinations that potentiate the antifungal fluconazole. Mol. Syst. Biol. 7:499. doi: 10.1038/msb.2011.31

Stern, M., Passweg, J. R., Meyer-Monard, S., Esser, R., Tonn, T., Soerensen, J., et al. (2012). Pre-emptive immunotherapy with purified natural killer cells after haploidentical SCT: a prospective phase II study in two centers. Bone Marrow Transplant. 48, 433-438. doi: 10.1038/bmt.2012.162

Stuehler, C., Nowakowska, J., Bernardini, C., Topp, M. S., Battegay, M., Passweg, J., et al. (2015). Multispecific aspergillus T cells selected by CD137 or CD154 induce protective immune responses against the most relevant mold infections. J. Infect. Dis. 211, 1251-1261. doi: 10.1093/infdis/jiu607

Sun, Z., Zhu, P., Li, L., Wan, Z., Zhao, Z., and Li, R. (2012). Adoptive immunity mediated by HLA-A*0201 restricted Asp f16 peptides-specific CD8 + T cells against Aspergillus fumigatus infection. Eur. J. Clin. Microbiol. Infect. Dis. 31, 3089-3096. doi: 10.1007/s10096-012-1670-2

Sutrave, G., Blyth, E., and Gottlieb, D. J. (2017). Cellular therapy for multiple pathogen infections after hematopoietic stem cell transplant. Cytotherapy 19, 1284-1301. doi: 10.1016/j.jcyt.2017.07.012

Tramsen, L., Koehl, U., Tonn, T., Latgé, J. P., Schuster, F. R., Borkhardt, A., et al. (2008). Clinical-scale generation of human anti-Aspergillus $\mathrm{T}$ cells for adoptive immunotherapy. Bone Marrow Transplant. 43, 13-19. doi: 10.1038/bmt. 2008.271

Tramsen, L., Schmidt, S., Boenig, H., Latgé, J.-P., Lass-Flörl, C., Roeger, F., et al. (2013). Clinical-scale generation of multi-specific anti-fungal T cells targeting Candida, Aspergillus and mucormycetes. Cytotherapy 15, 344-351. doi: 10. 1016/j.jcyt.2012.11.014

Tramsen, L., Schmidt, S., Roeger, F., Schubert, R., Salzmann-Manrique, E., Latgé, J.-P., et al. (2014). Immunosuppressive compounds exhibit particular effects on functional properties of human anti-aspergillus TH1 cells. Infect. Immun. 82, 2649-2656. doi: 10.1128/IAI.01700-14

Trevino-Rangel Rde, J., Villanueva-Lozano, H., Hernandez-Rodriguez, P., Martinez-Resendez, M. F., Garcia-Juarez, J., Rodriguez-Rocha, H., et al. (2016). Activity of sertraline against Cryptococcus neoformans: in vitro and in vivo assays. Med. Mycol. 54, 280-286. doi: 10.1093/mmy/myv109

Tzannou, I., Papadopoulou, A., Naik, S., Leung, K., Martinez, C. A., Ramos, C. A., et al. (2017). Off-the-shelf virus-specific T cells to treat BK virus, human herpesvirus 6, cytomegalovirus, epstein-barr virus, and adenovirus infections after allogeneic hematopoietic stem-cell transplantation. J. Clin. Oncol. 35, 3547-3557. doi: 10.1200/JCO.2017.73.0655

Valentini, C. G., Farina, F., Pagano, L., and Teofili, L. (2017). Granulocyte transfusions: a critical reappraisal. Biol. Blood Marrow Transplant. 23, 20342041. doi: 10.1016/j.bbmt.2017.07.029

van de Veerdonk, F. L., Kullberg, B. J., and Netea, M. G. (2012). Adjunctive immunotherapy with recombinant cytokines for the treatment of disseminated 
candidiasis. Clin. Microbiol. Infect. 18, 112-119. doi: 10.1111/j.1469-0691.2011. 03676.x

van Nood, E., Vrieze, A., Nieuwdorp, M., Fuentes, S., Zoetendal, E. G., De Vos, W. M., et al. (2013). Duodenal infusion of donor feces for recurrent Clostridium difficile. N. Engl. J. Med. 368, 407-415. doi: 10.1056/NEJMoa 1205037

Voigt, J., Hünniger, K., Bouzani, M., Jacobsen, I. D., Barz, D., Hube, B., et al. (2014). Human natural killer cells acting as phagocytes against Candida albicans and mounting an inflammatory response that modulates neutrophil antifungal activity. J. Infect. Dis. 209, 616-626. doi: 10.1093/infdis/ jit574

Wan, L., Zhang, Y., Lai, Y., Jiang, M., Song, Y., Zhou, J., et al. (2015). Effect of granulocyte-macrophage colony-stimulating factor on prevention and treatment of invasive fungal disease in recipients of allogeneic stem-cell transplantation: a prospective multicenter randomized phase IV trial. J. Clin. Oncol. 33, 3999-4006. doi: 10.1200/JCO.2014.60.5121

West, K. A., Gea-Banacloche, J., Stroncek, D., and Kadri, S. S. (2017). Granulocyte transfusions in the management of invasive fungal infections. Br. J. Haematol. 177, 357-374. doi: 10.1111/bjh.14597

Wiederhold, N. P., Patterson, T. F., Srinivasan, A., Chaturvedi, A. K., Fothergill, A. W., Wormley, F. L., et al. (2017). Repurposing auranofin as an antifungal: in vitro activity against a variety of medically important fungi. Virulence 8, 138-142. doi: 10.1080/21505594.2016.1196301

Wiseman, H., Cannon, M., and Arnstein, H. R. V. (1989). Observation and significance of growth inhibition of Saccharomyces cerevisiae (A224A) by the antioestrogen drug tamoxifen. Biochem. Soc. Trans. 17, 1038-1039. doi: 10 1042/bst0171038

Wright, C. R., Ward, A. C., and Russell, A. P. (2017). Granulocyte colonystimulating factor and its potential application for skeletal muscle repair and regeneration. Mediat. Inflamm. 2017:7517350. doi: 10.1155/2017/ 7517350

Wüthrich, M., Deepe, G. S., and Klein, B. (2012). Adaptive immunity to fungi. Annu. Rev. Immunol. 30, 115-148. doi: 10.1146/annurev-immunol-020711074958

Zhai, B., Wu, C., Wang, L., Sachs, M. S., and Lin, X. (2012). The antidepressant sertraline provides a promising therapeutic option for neurotropic cryptococcal infections. Antimicrob. Agents Chemother. 56, 3758-3766. doi: 10.1128/AAC. 00212-12

Conflict of Interest Statement: The authors declare that the research was conducted in the absence of any commercial or financial relationships that could be construed as a potential conflict of interest.

Copyright (c) 2018 Sam, Yew, Seneviratne, Chang and Chai. This is an open-access article distributed under the terms of the Creative Commons Attribution License (CC BY). The use, distribution or reproduction in other forums is permitted, provided the original author(s) and the copyright owner(s) are credited and that the original publication in this journal is cited, in accordance with accepted academic practice. No use, distribution or reproduction is permitted which does not comply with these terms. 